\title{
Point-of-Care Ultraschall in Notfallzentren
}

\section{Zielgerichtete Diagnostik zur raschen Therapieentscheidung}

Mathias Schmid, Christoph Dodt

Notfallzentrum München Klinik Bogenhausen

Bibliografie

10.1055/a-0803-8639

\section{ZUSAMMENFASSUNG}

Akut- und Notfallmediziner müssen mit wenigen diagnostischen Mitteln rasche und weitreichende Entscheidungen tref- fen. Im Rahmen einer Notfallversorgung ist der Point-of-Care Ultraschall (POCUS) als immer verfügbares, bettseitiges diagnostisches Hilfsmittel nicht mehr wegzudenken. Dabei geht es um eine fokussierte und symptomorientierte Untersuchung klar definierte Fragestellungen sollten durch den Notfallmediziner möglichst mit ja oder nein beantwortet werden. Durch immer kleinere „Handheld“-Ultraschallgeräte weitet sich der Einsatz des POCUS inner- und auch präklinisch aus.

\section{ABKÜRZUNGSVERZEICHNIS}

BLUE: Bedside Lung Ultrasound in Emergency COPD: Chronisch obstruktive Lungenerkrankung

FALLS: Fluid Administration Limited by Lung Sonography

FATE: Focus Assessed Transthoracic Echocardiography

ICR: Interkostalraum

LUS: Lungenultraschall

PLAPS: posterior und/oder lateral alveolar und/oder pleurales

Syndrom

POCUS: Point-of-Care Ultraschall

PW-Doppler: Pulsed-Wave-Doppler

RUSH: Rapid Ultrasound for Shock and Hypotension

SHoC: Sonography in hypotension and cardiac arrest

TTE: transthorakale Echokardiografie

ZVK: Zentraler Venenkatheter
Krankheitsbilder können durch ihn untersucht und abgeklärt werden. A Abb. 1 zeigt einen Ausschnitt der Einsatzmöglichkeiten [1]. Konventionelle Maßnahmen wie z. B. Röntgenbilder werden dadurch teilweise obsolet.

Anhand von Fallbeispielen und im Rahmen von Protokollen möchten wir Ihnen einige wichtige Aspekte und Fragestellungen des POCUS darlegen.

\section{Fall 1: Dyspnoe}

In der Notaufnahme wird ein Patient mit akuter Dyspnoe vorstellig. Es präsentiert sich ein hypertensiv entgleister, tachykard- und tachypnoeischer, zyanotischer agitierter 60-jähriger männlicher Patient. In der Vorgeschichte sind eine Herzinsuffizienz und eine COPD bekannt. Die vom Hausarzt verschriebenen Diuretika und Antiobstruktiva wurden in letzter Zeit nicht eingenommen.

Die akute Dyspnoe stellt ein häufiges Leitsymptom in der Notfallmedizin dar und ist mit einer hohen Sterblichkeit verbunden. Die Differenzialdiagnosen und Therapieansätze sind sehr unterschiedlich. Die Frage, ob der Patient unter einer kardialen Dekompensation mit pulmonaler Stauung, einer akut exazerbierten COPD, einem Pneumothorax oder eventuell einer Pneumonie leidet, kann in wenigen Minuten mit dem Lungenultraschall (LUS) gelöst werden.

Das BLUE-Protokoll (Bedside Lung Ultrasound in Emergency) wurde erstmalig von Lichtenstein beschrieben und stellt einen Algorithmus für Patienten mit akuter Luftnot dar. Je nach sonomorphologischer Darstellung der Pleura/Lunge wird gemäß dem BLUE-Protokoll jedem Befund ein Profil zugeordnet, welches dann hoch spezifisch für eine Diagnose (>90\%) ist [2]. So kann durch den LUS bei 


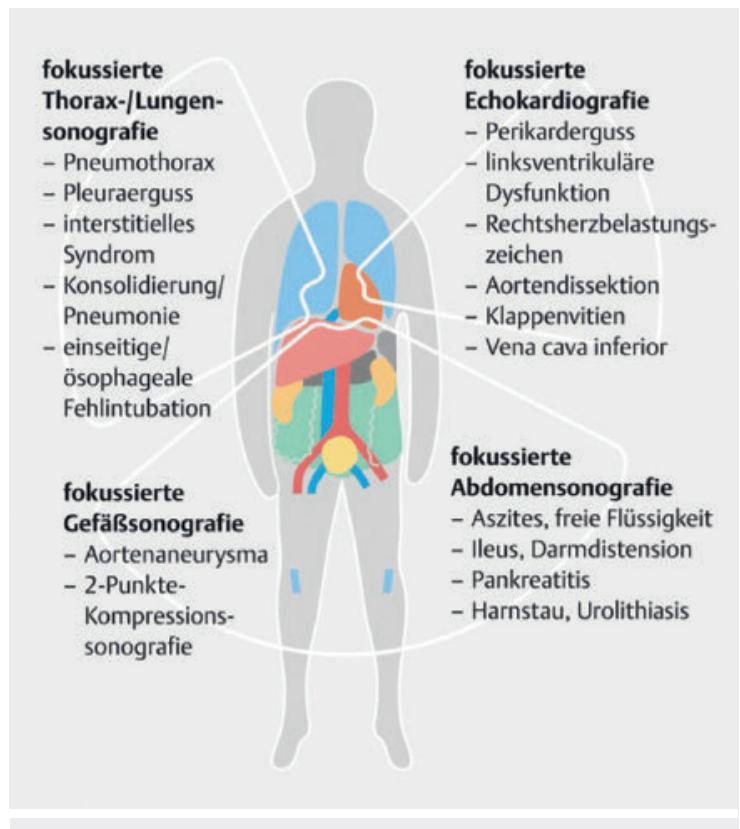

- Abb. 1 Ausschnitt der Einsatzmöglichkeiten des POCUS (aus [1]) [rerif].

unserem Patienten ein Asthma bronchiale/exazerbierte COPD gut von einem Asthma kardiale unterschieden werden. Dabei wird bei halbsitzendem bzw. liegendem Patienten mit dem Linearschallkopf (12-3 MHz) der ventrale Hemithorax an 2 „BLUE“-Punkten bilateral betrachtet (oberer BLUE-Punkt: medioklaviculär ca. 3. ICR, unterer BLUE-Punkt: vordere Axillarlinie ca. 5. ICR). Der Schallkopf wird senkrecht zu den Rippen angesetzt. Nun stellen sich am oberen Bildrand die obere und untere Rippe dar, dazwischen die Brustwand und die Pleuralinie. Die Pleurablätter bewegen sich normalerweise atemsynchron („Lungengleiten“). Beim Gesunden zeigen sich wiederholende, horizontale, echoreiche Linien unterhalb der Pleuralinie die sogenannten A-Linien. Der Abstand dieser Reverberationsartefakte entspricht genau der Distanz zwischen Pleura zum Schallkopf. Der Normalbefund des LUS, das sogenannte A-Profil, definiert sich über ein Lungengleiten mit vorherrschenden horizontalen A-Linien ( $\mathbf{A} \mathbf{b b}$. 2a).

Bei dem Patienten im Fallbeispiel zeigen sich bilateral an den BLUE-Punkten mehrere vertikale, laserartige, echoreiche Artefakte, die der Pleuralinie entspringen, die ALinien überlagern und bis an den unteren Bildrand gehen die B-Linien ( $\triangleright$ Abb. $\mathbf{2 b}$ ). Sie erinnern an einen Kometenschweif und werden daher auch als "comet-tail artifacts“ bezeichnet. Der bilaterale Nachweis von $\geq 3$ B-Linien in einem ICR und an jeweils 2 BLUE-Punkten der anteriolateralen Thoraxwand ist hochspezifisch (95\%) und sensitiv (97\%) für eine pulmonale Stauung - es liegt dann ein B-Profil vor [2]. Der LUS ist bei der Diagnosestellung einer dekompensierten Herzinsuffizienz dem Röntgen-Thorax, aber auch der klinischen Untersuchung, der Anamnese und dem EKG überlegen [3]. Jedoch sollte natürlich der LUS in
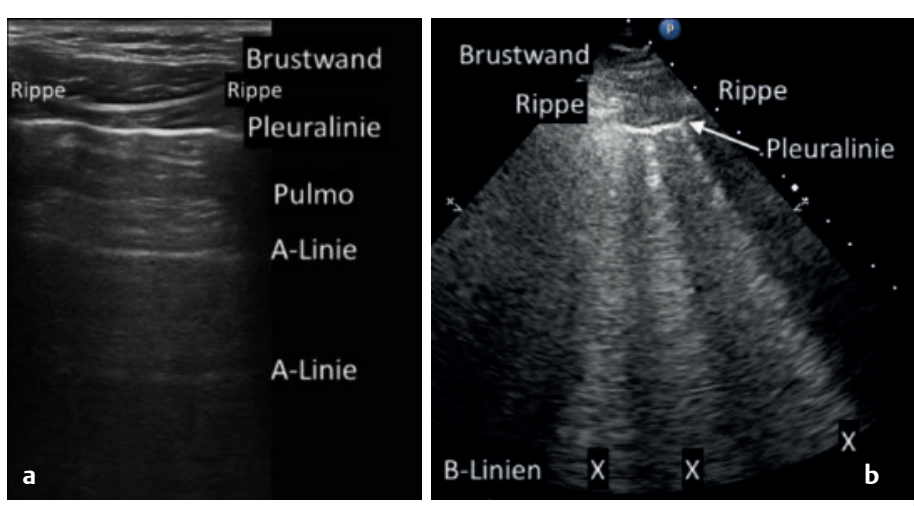

- Abb. 2 a Normalbefund LUS (B-Bild) mit dem Linearschallkopf. b 3 typische B-Linien ( $\mathrm{x}$ ) an der anterioren Thoraxwand mit dem Sektorschallkopf. [rerif]

Zusammenschau aller Informationen betrachtet werden der POCUS ist ein zunehmend wichtiger Baustein.

Weiterhin wird bei unserem Patienten auch ein dorsolateraler Punkt, der sogenannte PLAPS-Punkt (posterior und/ oder lateral alveolar und/oder pleurales Syndrom) angelotet. Dieser stellt den costophrenischen Winkel dar. Hier zeigt sich beidseits ein echofreier Pleurarandwinkelerguss.

Somit ist die Diagnose bei dem Patienten im Fallbeispiel eine (hypertensiv) entgleiste Herzinsuffizienz mit Lungenödem - die entsprechende Therapie kann innerhalb von Minuten nach Eintreffen eingeleitet werden.

Um die diagnostische Sicherheit bei vermuteter Herzinsuffizienz zu erhöhen, kann - wenn zeitlich möglich - eine orientierende Echokardiografie sowie die Betrachtung der Vena cava inferior erfolgen (siehe auch Fall 2) [4]. Weiterhin können durch den LUS eine Pneumonie oder ein Pneumothorax abgegrenzt werden. Nach internationalen Empfehlungen ist der LUS inzwischen das primäre Diagnostikum bei V. a. Lungenödem [5].

\section{Fall 2a: Schock}

Die Behandlung von Patienten im Schock stellt eine Herausforderung dar und bedarf einer raschen Diagnostik mit daraus resultierender Therapieentscheidung. Für die zeitnahe Einschätzung der Schockursache existieren viele Ultraschallprotokolle (u. a. RUSH, FALLS, FATE). 2017 wurde ein internationaler Konsens zum POCUS bei Patienten im Schock bzw. bei Reanimation publiziert [6].

Ein 75-jähriger Patient wird bei thorakalen Schmerzen mit V. a. ein akutes Koronarsyndrom vorstellig. Im EKG zeigen sich keine ischämietypischen Veränderungen, der Kreislauf ist stabil, seine Beschwerden sind auf Analgetika und NitroSpray rückläufig. Während der Monitorüberwachung bei initial leicht erhöhtem Troponin I krampft der Patient tonisch-klonisch. Auffallend sind danach eine Hypotension bei Normofrequenz (Cave: Vortherapie mit $\beta$-Blockern), 

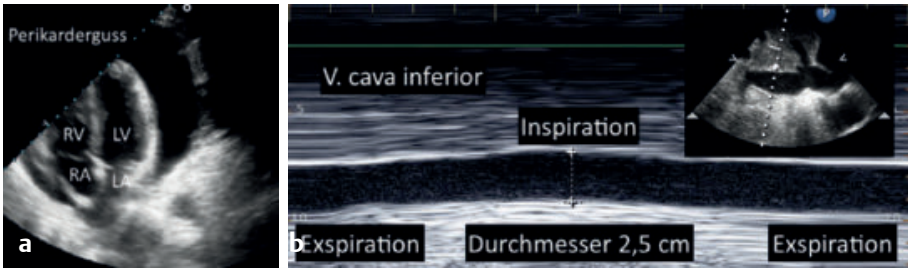

- Abb. 3 a Von subxiphoidal erkennbarer, großer zirkumferenter Perikarderguss, echofrei. $\mathbf{b}$ durch Kippung und Drehung des Schallkopfes sichtbare V. cava inf. in der Längsachse, diese im M-Mode weit $(2,5 \mathrm{~cm})$ und nicht atemmoduliert. [rerif]

eine persistierende Vigilanzminderung, eine deutlich verlängerte Rekapillarisierungszeit und eine Marmorierung beider Knie.

Gerade bei Patienten im Schock ist der POCUS unersetzbar, besonders hier sollten Ja-/Nein-Fragen sofort beantwortet werden. Wir gehen bei unserem Patienten nach dem SHoC-Hypotension-Protokoll (Sonography in hypotension and cardiac arrest) gemäß dem internationalen Konsens vor [6]. Zunächst wird das Herz von subxiphoidal oder der langen parasternalen Achse betrachtet. Fragestellungen sind: Perikarderguss/-tamponade, ventrikuläre Pumpfunktion, Beurteilung der Größe der Herzhöhlen. Durch einen Schwenk des Schallkopfes nach kaudal und Drehung um $90^{\circ}$ wird die Vena cava inferior im Längsschnitt beurteilt (möglichst im M-Mode mit Betrachtung der Atemvariabilität). Danach erfolgt der LUS analog dem BLUE-Protokoll. In vielen Fällen steht die Schockursache nach diesen ersten Untersuchungen fest und die zielgerichtete Therapie kann eingeleitet werden. Durch diesen ersten Untersuchungsschritt sollte auch der Volumenstatus des Patienten eingeschätzt werden können. Weitere Informationen können im nächsten Schritt durch die orientierende Echokardiografie durch Anlotung der kurzen parasternalen Achse und von apikal ergänzt werden. Je nach initialer Verdachtsdiagnose, z. B. dem V.a. einen hypovolämen Schock (Cava: schlanke, atemmodulierte V. cava inferior; TTE: hyperkontraktile, „leer“ pumpende Ventrikel; LUS: A-Profil), wird weiter nach einer intraabdominellen Blutung gesucht. Bei akuten Rechtsherzbelastungszeichen (V. a. Lungenarterienembolie) kann ein Kompressionsultraschall der proximalen Beinvenen die Diagnose sichern (Thrombose?). Es wird jedoch insbesondere in der internationalen Empfehlung betont, dass der POCUS beim Schock nicht starr nach Protokoll erfolgen soll, sondern die klinisch wahrscheinlichste Diagnose zuerst sonografisch abzuklären ist, also eine an Symptom- und Verdachtsdiagnosen orientierte Untersuchung priorisiert wird - daher auch der hierarchische Untersuchungsablauf des SHoC-Hypotension-Protokolls [6].

Bei dem Fallbeispiel wurde mit dem Sektorschallkopf (5-1 $\mathrm{MHz}$ ) das Herz von subxiphoidal angelotet und es zeigt sich ein großer, zirkumferenter Perikarderguss, die rechten Herzhöhlen sind kollaptisch ( $\triangleright$ Abb. 3a). Beim Blick auf die V. cava inferior stellt sich diese $2,5 \mathrm{~cm}$ weit und nicht atem- moduliert dar ( $\triangleright$ Abb. 3b). Somit liegt in Zusammenschau mit der Klinik eine Perikardtamponade vor. Der Ultraschall ist das Diagnostikum der Wahl bei V. a. eine Perikardtamponade. Es sollte sofort eine sonografisch gesteuerte Perikardpunktion erfolgen [7, 8]. Nach Perikardpunktion und Ablassen von $250 \mathrm{ml}$ Blut stabilisierte sich der Kreislauf unseres Patienten rasch. In der CT-Aortografie wurde eine Aortendissektion Stanford A mit Einriss der Aorta bis in das Perikard diagnostiziert.

\section{Fall 2b: Reanimation}

Ein Patient kommt unter laufender Reanimation in den Schockraum. Das Beschwerdebild und die Klinik vor Reanimation gleichen denen des Patienten mit Perikardtamponade (Fall 2a). Einige der HITS/4H's können mittels POCUS unmittelbar abgeklärt werden, jedoch dürfen durch die Sonografie keinesfalls „no flow“-Zeiten entstehen. Laut ERC-Leitlinien soll der POCUS während der Rhythmusanalyse innerhalb von 10 sec durchgeführt werden [9]. Daher sind eine gute Vorbereitung und Teamabsprache wichtig. Wir gehen nach dem SHoC-Reanimation-Protokoll der o.g. internationalen Konsensusgruppe vor [6]. Der Sektorschallkopf wird subxiphoidal (ggf. parasternal) angesetzt, während der Rhythmusanalyse das Herz angelotet und ein Video aufgezeichnet - im Anschluss wird dieses unter Fortführung der Reanimation ausgewertet. Eine Herzbeuteltamponade, eine Rechtsherzbelastung (Thrombose) sowie die Pumpfunktion (Asystolie vs. organisierte Herzaktivität) sollte bewertet werden können. Erweitert wird die Lunge (Spannungspneumothorax) und die V. cava inferior (Hypovolämie) geschallt. Danach kann je nach Fragestellung u. a. auch die Tubuslage beurteilt und in Analogie zum SHoC-Hypotension-Protokoll nach einer tiefen Venenthrombose oder einem Blutverlust (Hypovolämie) gesucht werden.

Während der Rhythmusanalyse stellt sich bei unserem Patienten von subxiphoidal ein deutlich vergrößerter rechter Ventrikel mit schmaler Ventrikelwand im Sinne einer akuten Rechtsherzbelastung dar ( $\mathbf{A b b}$. 4a). Im Beinvenen-Kompressionsultraschall zeigt sich linksseitig eine Thrombose der V. femoralis superficialis ( $\mathbf{A} \mathbf{b} \mathbf{b}$. $\mathbf{4 b}$, Abb. 4c). Somit ist eine Lungenarterienembolie als Reanimationsursache hochwahrscheinlich - die Therapie kann auf Basis des POCUS umgehend begonnen werden.

\section{Fall 3: Bauchschmerzen}

Eine 23-jährige Patientin ohne Vorerkrankungen stellt sich mit seit 2 Tagen bestehenden abdominellen Schmerzen vor, der p.m. sei immer im rechten Unterbauch gewesen. Es bestünden subfebrile Temperaturen $\left(37,4^{\circ} \mathrm{C}\right)$, etwas Übelkeit ohne Erbrechen, 2 x weicherer Stuhlgang. Laborwerte: Leukozytose 12,4/nl mit Linksverschiebung, CRP 25 mg/l (Normalwert $<5 \mathrm{mg} / \mathrm{l}$ ), Schwangerschaftstest negativ. In der klinischen Untersuchung findet sich eine Abwehrspannung im 

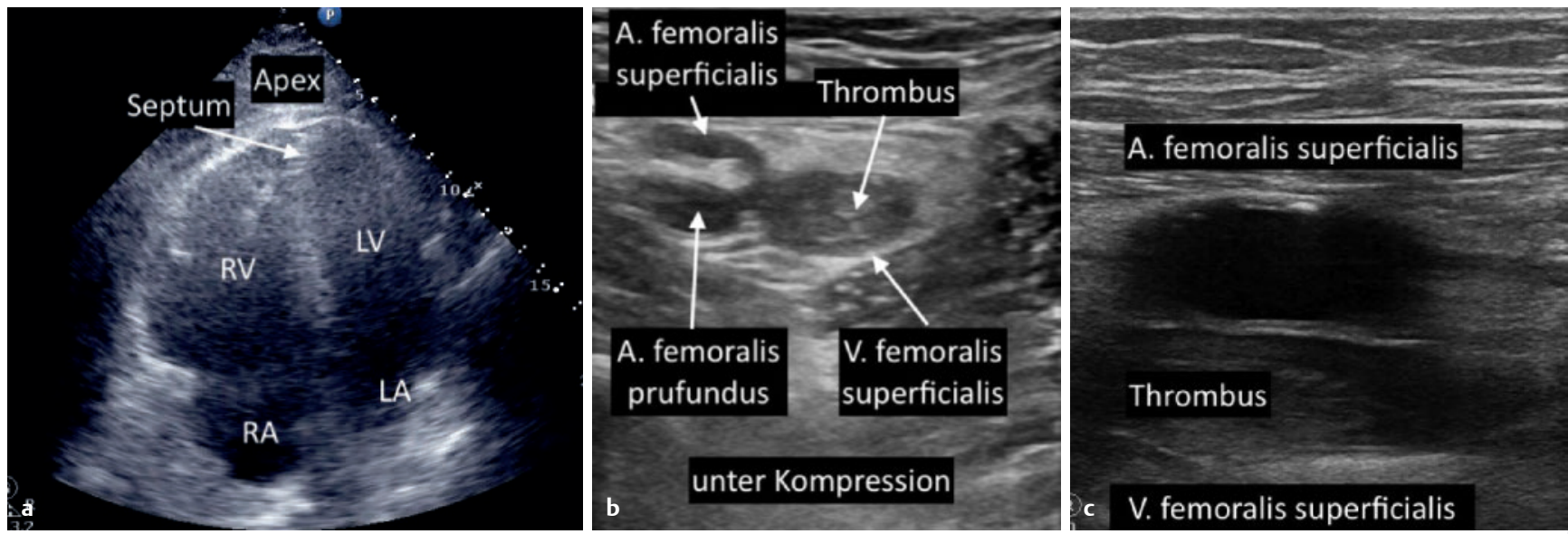

- Abb. 4 a Subxiphoidale Anlotung: Der rechte Ventrikel (RV) ist deutlich größer und balloniert im Vergleich zum Linken (LV); (RA/LA: rechter/linker Vorhof). b Kompressionsultraschall linker Oberschenkel im Quer- bzw. c Längsschnitt. Die V. femoralis superficialis ist nicht komprimierbar. [rerif]

rechten Unterbauch (McBurney-Punkt) mit kontralateralem Loslassschmerz (Blumberg-Zeichen). Die Anamnese und Klinik weisen deutlich auf eine Appendizitis hin.

Patienten suchen oft wegen abdomineller Schmerzen die Notaufnahmen auf. Die akute Appendizitis ist bei einer Lebenszeitindizienz von 6-9\% die häufigste Ursache für ein akutes Abdomen. Die Frühzeichen einer Appendizitis sind jedoch oft subtil [10]. Bei der Patientin im Fallbeispiel beträgt der Alvarado-Score 8 (mittlere Wahrscheinlichkeit). Nach den Leitlinien der europäischen Chirurgen wird im weiteren Schritt eine Bildgebung gefordert, insbesondere um die Rate unnötiger Appendektomien, die um die 15\% liegt, zu reduzieren [11]. Primär sollte eine abdominelle Sonografie erfolgen. In einer Metaanalyse beträgt die Sensitivität des Ultraschalls $91 \%$, die Spezifität $97 \%$. Der positive Likelihoodquotient ist hoch (6-46), jedoch der Negative nur moderat gut $(0,08-0,3)$. Somit eignet sich der Ultraschall gut für die Bestätigung einer Appendizitis, jedoch ist der Ausschluss nicht unbedingt möglich. Daher sollte sich bei nicht eindeutigem Sonografiebefund (je nach Alvarado-Score) eine weitere Bildgebung (CT oder MRT) anschließen [11].

Die sonografische Darstellung der Appendix fordert Übung. Mit dem Konvexschallkopf (5-2 MHz) wird mit der Untersuchung entweder am Punctum maximum des Schmerzes begonnen oder das Colon ascendens von kranial nach kaudal im Längsschnitt bis zum lleozökalpol verfolgt [7, 12]. Durch sanften Druck wird Darmgas verdrängt und der Dünndarm komprimiert. Üblicherweise liegt die intraperitoneal lokalisierte Appendix an der Medialseite des Ileozökalpols, seltener retrozökal. Sie erscheint im Normalbefund im Längsschnitt als tubuläre, blind endende Struktur. Eine Peristaltik ist im Gegensatz zum Dünndarm nicht vorhanden, normalerweise ist sie komprimierbar.

Der Schmerzpunkt unserer Patientin lässt sich gut lokalisieren, im Linearschallkopf (12-3 MHz) sehen wir eine deutlich verdickte Appendix (Grenzwert im Querdurch-
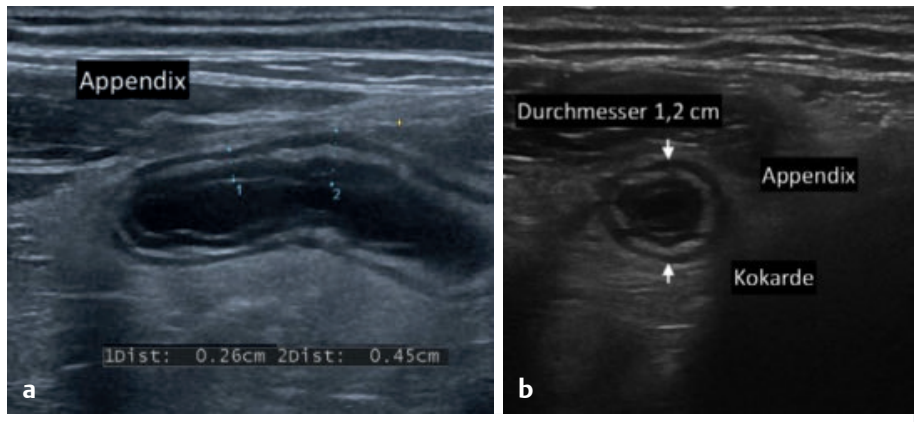

Abb. 5 a Typische Appendizitis mit deutlich vergrößerter Appendix und aufgetriebener Wand im Längsschnitt. b Kokarde im Querschnitt $(1,2 \mathrm{~cm}$ Durchmesser). [rerif]

messer 6-8 mm) mit prominenter Wand, bei Palpation der Struktur mit dem Schallkopf verstärkt sich der Schmerz (Zeichen einer lokalen Peritonitis, \ Abb. 5a). Oft entsteht im Querschnitt die typische Kokarde ( $\bullet$ Abb. 5b). Um die echoarme Flüssigkeit im Lumen schließen sich ringartig die echoreiche Mucosa, Submucosa und Serosa, die jeweils durch eine echoarme Muskelschicht getrennt sind. Dadurch ist die Appendixwand aufgetrieben und verdickt (>2 mm). Manchmal ist zentral ein echoreicher Appendikolith mit dorsalem Schallschatten darstellbar. Fortgeschrittene Entzündungen können zu lokalen Flüssigkeitsansammlungen und Abszedierungen führen. Differenzialdiagnostisch sind sonografisch u. a. die Enteritis, Divertikulitis, Nierenkolik, der lleus und besonders auch bei Patientinnen die Adnexitis abzugrenzen.

Bei der Patientin im Fallbeispiel ist mit einem intermediären Alvarado-Score (8 Punkte) und einem positiven Ultraschallbefund eine Appendizitis bewiesen [11].

\section{Fall 4: Interventionen}

Ein Patient mit septischem Schock wird durch den Rettungsdienst eingeliefert. Der Infektfokus ist anamnes- 

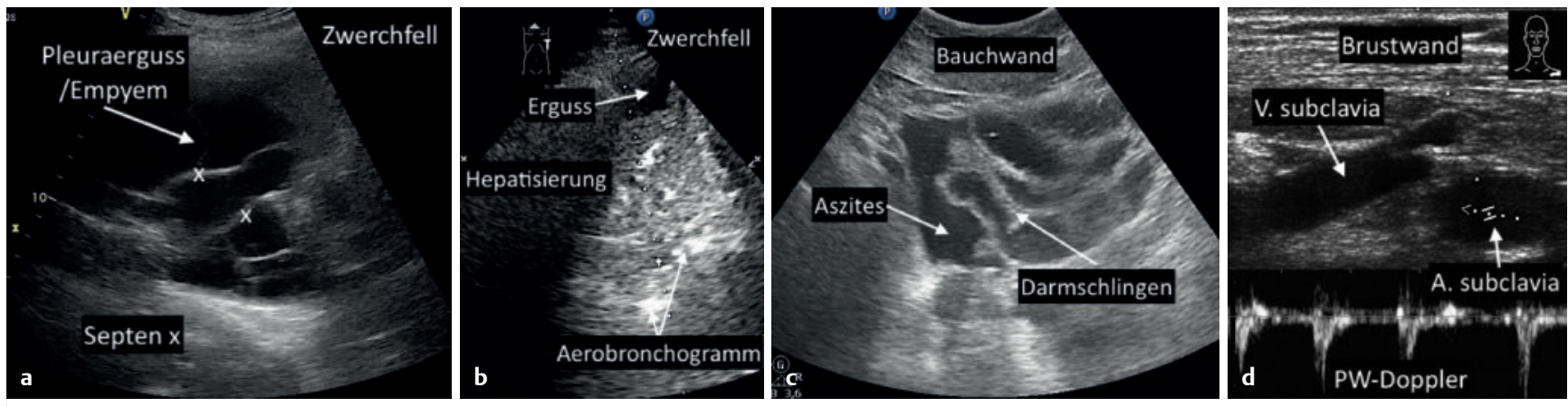

- Abb. 6 a Pleuraerguss linksseitig mit Septen, DD. Pleuraempyem. b weiter dorsal Hepatisierung der Lunge mit Aerobronchogramm. c Aszites im rechten Unterbauch, Dünndarmschlingen „schwimmen“ in diesem. d Anlotung der V. subclavia links im Längsschnitt mit PW-Doppler in der A. subclavia. [rerif]

tisch unklar, im LUS zeigt sich linksseitig ventrokaudal ein großer, septierter Pleuraerguss ( $>$ Abb. 6a), weiter dorsal eine Hepatisierung der Lunge mit Aerobronchogramm im Sinne einer Pneumonie ( $\triangleright$ Abb. 6b). Weiterhin stellt sich im abdominellen Ultraschall lokaler Aszites im rechten Unterbauch dar ( $\triangleright$ Abb. 6c). Bei unzureichendem Venenstatus und septischem Schock sind zur Volumen- und Katecholamintherapie ein ZVK und eine Arterie notwendig. Bei einer Vielzahl von Eingriffen kann die Sonografie bei niedriger Komplikationsrate die Erfolgsrate erhöhen. Es wird empfohlen die ZVK-Anlage (weniger Komplikationen, schnellere Anlage, Kostenreduktion), die diagnostische/therapeutische Pleurapunktion, die Aszitespunktion und die schwierige periphere arterielle und venöse Katheterisierung sonografisch gesteuert durchzuführen [8, 13, 14]. Besonders bei hypovolämen Patienten (z. B. beim septischen Schock) ist die Anlage eines ZVK oft schwierig und Komplikationen sind nicht selten. Zunächst wird sonografisch gesteuert die linke V. subclavia punktiert und ein ZVK in Seldinger-Technik gelegt ( $\triangleright$ Abb. $6 \mathbf{d}$ ). Bei septischem Schock und o.g. Pleuraerguss muss ein Pleuraempyem ausgeschlossen werden. Unter empfohlener Ultraschallkontrolle (Pneumothoraxrate unter Ultraschallsteuerung deutlich vermindert) wird eine $14 \mathrm{Fr}$ Minidrainage in Seldinger-Technik eingebracht. Darüber entleert sich ein parapneumonischer Erguss (hell-gelblich, nicht eitrig, pH 7,1). Im Anschluss erfolgt ein LUS, an den ventralen BLUE-Punkten zeigt sich auf der Punktionsseite Lungengleiten, somit ist ein Pneumothorax nach ZVK-Anlage und Pleurapunktion links unwahrscheinlich. Weiterhin wird eine ultraschallgesteuerte Aszitespunktion zum Ausschluss einer Peritonitis durchgeführt (heller Aszites, niedrige Zellzahl). Die arterielle Kanülierung erfolgt gewöhnlich ohne Ultraschall, obwohl dies empfohlen wird [8]. Mithilfe des POCUS liegen sichere Zugänge, als Sepsisursache wurde eine Pneumonie diagnostiziert, ein parapneumonischer Erguss behandelt und eine Peritonitis ausgeschlossen. Beim Volumenmanagement hat der POCUS initial wie auch im Verlauf einen wichtigen Stellenwert.

Die beschriebenen Fallbeispiele und Pathologien stellen nur einen Ausschnitt der Indikationen und Möglichkei- ten des POCUS dar. Ebenso sind die jeweiligen erwähnten Protokolle nur als eine Option der Herangehensweise anzusehen. Hervorzuheben ist noch die fokussierte Sonografie der Bewegungsorgane. Sie ermöglicht Befunde (Fraktur/Fissur, Luxation, Erguss, Ruptur, Fremdkörper) mit guter diagnostischer Genauigkeit zu erheben. Besonders Flüssigkeitsansammlungen um Sehnen, Schleimbeutel oder im Bindegewebe sowie Gelenkergüsse sind einfach zu detektieren. Je nach Erfordernis können weitere bildgebende Verfahren indiziert sein oder eine (Ab-)Punktion mit Diagnostik erfolgen. Diesbezüglich dürfen wir auf weiterführende Literatur verweisen [15].

Der POCUS kann sowohl in Büchern als auch online (mit reichlich Videomaterial) weiter vertieft werden, siehe $\checkmark$ Infobox.

\section{Fazit für die Praxis}

- Der POCUS gewinnt mit der Verbreitung von mobilen und „Handheld“-Ultraschallgeräten zunehmend Bedeutung in der Notfalldiagnostik, die Indikationen sind weitreichend und werden weiter zunehmen.

- Ziel des POCUS ist eine fokussierte und symptomorientierte Untersuchung.

- Der Notfallmediziner soll klar definierte Fragestellungen möglichst mit ja oder nein beantworten können.

- Durch den POCUS lässt sich umgehend die diagnostische Sicherheit lebensbedrohlicher Krankheitsbilder wie Dyspnoe und Schock mithilfe von Protokollen verbessern und die zielgerichtete Therapie einleiten.

- Das BLUE-Protokoll ist ein bewährter Algorithmus bei Patienten mit akuter Dyspnoe.

- Für Patienten im Schock existieren viele Protokolle, international wird das SHoC-Hypotension-Protokoll empfohlen.

- Durch den POCUS der Bewegungsorgane können wichtige Befunde (Fraktur/Fissur, Luxation, Erguss, Ruptur, Fremdkörper) mit guter diagnostischer Genauigkeit erhoben werden.

- Zur Vertiefung des Themas können im Internet Tutorials mit Videos abgerufen werden (siehe $>$ Infobox). 
INFOBOX: MEHR INFORMATIONEN ZUM POCUS

- Buch: Lichtenstein D. Lung ultrasound in the critically ill : the BLUE protocol. Cham: Springer; 2016

- Buch: Ma OJ. Ma and Mateer‘s emergency ultrasound. 3 rd. Aufl. New York: McGraw-Hill Education Medical; 2014

- Webpage: http://5 minsono.com

- Webpage: http://www.emergencyultrasoundteaching.com/index.html

- Webpage: http://www.thepocusatlas.com/

- Twitter: \#pocus

\section{Interessenkonflikt}

Die Autoren geben an, dass kein Interessenkonflikt besteht.

\section{Einhaltung ethischer Richtlinien}

Dieser Beitrag beinhaltet keine Studien an Menschen oder Tieren.

\section{Danksagung}

Die Autoren danken Frau Dr. Melanie Schmid und Herrn Dr. Niklas Schneider für die hilfreiche Unterstützung.

\section{Korrespondenzadresse}

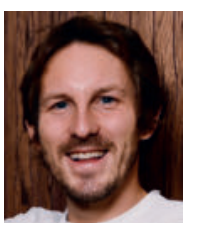

\section{Dr. med. Mathias Schmid}

Oberarzt Notfallzentrum

München Klinik Bogenhausen

Englschalkinger Str. 77

81925 München

Mathias.Schmid@klinikum-muenchen.de official journal of the Society for Academic Emergency Medicine 2016; 23: 223-242

[4] Ohman J, Harjola VP, Karjalainen P et al. Focused echocardiography and lung ultrasound protocol for guiding treatment in acute heart failure. ESC heart failure 2018; 5: 120-128

[5] Volpicelli G, Elbarbary M, Blaivas M et al. International evidence-based recommendations for point-of-care lung ultrasound. Intensive care medicine 2012; 38: 577-591

[6] Atkinson P, Bowra J, Milne J et al. International Federation for Emergency Medicine Consensus Statement: Sonography in hypotension and cardiac arrest (SHoC): An international consensus on the use of point of care ultrasound for undifferentiated hypotension and during cardiac arrest. Cjem 2017; 19: 459-470

[7] Ma OJ. Ma and Mateer's emergency ultrasound. 3 rd. Aufl. New York: McGraw-Hill Education Medical; 2014

[8] Lamperti M, Bodenham AR, Pittiruti M et al. International evidence-based recommendations on ultrasound-guided vascular access. Intensive care medicine 2012; 38: 1105-1117

[9] Soar J, Nolan JP, Böttiger BW et al. Erweiterte Reanimationsmaßnahmen für Erwachsene („adult advanced life support“). Notfall + Rettungsmedizin 2015; 18: 770-832

[10] Matthew Fields J, Davis J, Alsup C et al. Accuracy of Point-ofcare Ultrasonography for Diagnosing Acute Appendicitis: A Systematic Review and Meta-analysis. Academic emergency medicine : official journal of the Society for Academic Emergency Medicine 2017; 24: 1124-1136

[11] Gorter RR, Eker HH, Gorter-Stam MA et al. Diagnosis and management of acute appendicitis. EAES consensus development conference 2015. Surgical endoscopy 2016; 30 : 4668-4690

[12] Kaneko T, Heinz W. [Emergency ultrasound in patients with abdominal pain - where should we „look“]. Deutsche medizinische Wochenschrift 2015; 140: 1606-1609

[13] Moore CL, Copel JA. Point-of-care ultrasonography. The New England journal of medicine 2011; 364: 749-757

[14] Frankel HL, Kirkpatrick AW, Elbarbary M et al. Guidelines for the Appropriate Use of Bedside General and Cardiac Ultrasonography in the Evaluation of Critically III Patients-Part I: General Ultrasonography. Critical care medicine 2015; 43: 2479-2502

[15] Horn R. [Focused musculoskeletal sonography]. Praxis (Bern 1994) 2015; 104: 1027-1032

Literatur

[1] Busche C, Busch H], Michels G. [Point-of-Care Sonography in Emergency and Intensive Care Medicine]. Deutsche medizinische Wochenschrift 2018; 143: 161-164

[2] Lichtenstein DA, Meziere GA. Relevance of lung ultrasound in the diagnosis of acute respiratory failure: the BLUE protocol. Chest 2008; 134: 117-125

[3] Martindale JL, Wakai A, Collins SP et al. Diagnosing Acute Heart Failure in the Emergency Department: A Systematic Review and Meta-analysis. Academic emergency medicine: 